\begin{tabular}{|l|l|l|l|l|l}
\hline Intropica & ISSN 1794-161X & Vol. 13 & No.1 & $21-29$ & Santa Marta-Colombia, enero-junio de 2018 \\
\hline
\end{tabular}

\title{
CARACTERIZACIÓN MORFOMÉTRICA DE UNA LAGUNA COSTERA NEOTROPICAL (CIÉNAGA EL CHINO, MAGDALENA - COLOMBIA)
}

\author{
NEOTROPICAL COASTAL LAGOON'S MORPHOMETRIC CHARACTERIZATION \\ (CIÉNAGA EL CHINO, MAGDALENA - COLOMBIA)
}

Jaime de Jesús Rodríguez Curcio@, Jean Linero-Cueto@ y Leandro José Barros Ortiz (iD

\begin{abstract}
RESUMEN
La morfometría de lagunas es esencial para la gestión de los recursos acuáticos y los ecosistemas limnéticos. En sistemas lacustres que reciben altas cargas de sedimentos, es necesario un mapeo morfométrico frecuente para definir tanto la vida efectiva como su capacidad de almacenamiento y control de inundaciones, entre otros. Se realizó un levantamiento batimétrico (2015 y 2017) en la ciénaga costera Neotropical El Chino (costado oriental de la Ciénaga Grande de Santa Marta) con el fin de conocer la distribución de profundidades y determinar los principales parámetros morfométricos. Se encontró un cuerpo de agua somero e hipersalino, con un perímetro rodeado por una espesa franja manglar e islotes de esta misma vegetación en algunas zonas. El cuerpo de agua mostró una profundidad máxima de 0,67 y 0,77 m, profundidad relativa muy baja $(0,0201$ y 0,0292), área de $8,62 \mathrm{~km}^{2}$, volumen de $4,30 \mathrm{Mm}^{3}$ y 5,248 $\mathrm{Mm}^{3}$, en 2015 y 2017, respectivamente. Longitud máxima de 5,89 km y orientado en la dirección Sur-suroeste (SSO) - Nor-noreste (NNE), ancho máximo de 2,14 km. El factor de forma indica que es un cuerpo de agua de forma cóncava, la pendiente promedio es muy baja (0,0974 \%). La relación Zm:Z > 0,4 indica que puede presentar mayores riesgos de colmatación. Es un cuerpo de agua muy inestable, no se observaron estratos de temperatura y que de llegar a existir serían inmediatamente perturbados por cualquier agente externo. En 2015 se encontró un ecosistema afectado por el fenómeno de El Niño. Los dos periodos de estudio mostraron un sistema con muchos parámetros similares, y la época de lluvias en 2017 no mejora ostensiblemente algunos de ellos, que son indicativos de la salud de la ciénaga El Chino. Otros impactos ambientales pudieron deberse a las actividades antrópicas que modifican la hidráulica natural del sistema.
\end{abstract}

PALABRAS CLAVE: morfometría; ciénaga; mapa batimétrico; laguna costera; departamento del Magdalena

\begin{abstract}
Lagoon morphometry is essential for managing water resources and limnetic ecosystems. In lacustrine systems that receive high sediment loads, a frequent morphometric mapping is necessary to define both the effective life and its water storage capacity and flood control, among others. A bathymetric survey (2015 and 2017) was developed in Neotropical coastal lagoon El Chino (eastern side of the Ciénaga Grande de Santa Marta) in order to know the distribution of depths and determine the main morphometric parameters. A very shallow and hypersaline waterbody was found. It has a perimeter surrounded by a thick mangrove strip and islets with same vegetation in several areas. This wetland has maximum depth of $0.67 \mathrm{~m}$ and 0.77 $\mathrm{m}$, very low relative depth (0.0201 and 0.0292), area of $8.62 \mathrm{~km}^{2}$, volume of $4.30 \mathrm{Mm}^{3}$ and $5.248 \mathrm{Mm}^{3}$, in 2015 and 2017 , respectively. Maximum length $5.89 \mathrm{~km}$, towards South-southwest (SSO) - Nor-northeast (NNE), maximum width of $2.14 \mathrm{~km}$. Shape factor indicates a concave waterbody, the average slope is very low $(0.0974 \%)$. The ratio $\mathrm{Zm:Z}>0.4$ indicates that El Chino swamp could have a great risk of silting. It is a very unstable waterbody, it does not have temperature strata and, if it came to exist, it would be immediately disturbed by any external force. In 2015 an ecosystem affected by El Niño phenomenon was found. Both periods show a system with very similar parameters, and the rainy season in 2017 does not improve some of them, which are health indicators of Chino Swamp. Other environmental impacts may be due to anthropogenic activities that modify its natural hydraulic.
\end{abstract}

KEY WORDS: morphometry; swamp; bathymetric chart; coastal lagoon; department of Magdalena

Dirección de los autores

Grupo de investigación en biodiversidad y conservación de ecosistemas. Fundación para la Participación Capacitación y la Investigación Social "FUPARCIS”, Calle 25 \# 2-124, Santa Marta, Colombia; email: jaimercurcio@gmail.com (J.J.R.C); eng.leandrobarros@yandex.com (L.J.B.O). Universidad del Magdalena. Carrera 32 No 22 -08 Santa Marta, Colombia; email: linero@unimagdalena.edu.co (J.L.C). 


\section{INTRODUCCIÓN}

Las ciénagas son ecosistemas muy dinámicos que desempeñan varias funciones ambientales, como control de inundaciones, recarga de acuíferos y retención de nutrientes y sedimentos. En Colombia estos cuerpos de agua, normalmente, no superan los cinco metros de profundidad y están localizados por debajo de los 1000 msnm (Benjumea Hoyos et al., 2010). La batimetría es necesaria para la descripción de los principales parámetros morfométricos (Benjumea Hoyos et al., 2010).

En el caso de ecosistemas lénticos, la morfometría comprende el conjunto de métodos para medir las dimensiones físicas de un sistema (Roldán y Ramírez, 2008). Idealmente, la caracterización morfométrica de un cuerpo de agua debe ser el punto de partida de las investigaciones limnológicas (Guiral y Pérez, 1980; Rico et al., 1995; Montoya Moreno, 2005). Uno de los principios limnológicos más antiguos es que la morfometría de la cuenca influye en el metabolismo de lagunas. Diferentes estudios han demostrado que ciertos procesos limnológicos y la distribución de parámetros físicos, biológicos y químicos están ligados directa o indirectamente a la morfometría y al periodo de retención de agua de este tipo de cuerpos de agua (Montoya Moreno, 2005; Roldán y Ramírez, 2008). Håkanson (2005) asegura que la forma y el tamaño de los lagos regulan procesos de transporte en general, lo cual justifica el estudio de estos cuerpos de agua.

En reservorios tropicales de Latinoamérica hay pocos estudios sobre parámetros morfométricos (Aldama et al., 2013). A nivel nacional se han realizado pocos estudios morfométricos dentro de los que se destacan los trabajos hechos en embalses por Hernani y Ramírez (2002); en lagos los realizados por Montoya Moreno (2005) y Rivera et al. (2010); y en ciénagas Benjumea Hoyos et al., (2010), Montoya Moreno (2008) y Montoya et al. (2011); brindando elementos que permiten comparar sistemas lénticos de zonas templadas con los someros lacustres.

En el norte de Colombia no se encuentran trabajos desarrollados en temas de morfometría en las ciénagas. Sin tener en cuenta todos los estudios desarrollados en la Ciénaga Grande de Santa Marta, estos van enmarcados en diferentes temas, dentro de los que, a manera de ejemplo, podemos mencionar los desarrollados por Zapata Gaviria (2005); Restrepo et al. (2006); Gómez
Rivera (2008); Hernández-Atilano et al. (2008); MolanoRendón et al. (2008); Montoya y Aguirre (2009); JiménezSegura et al. (2010); Castro Rodríguez (2012); Rúa-García (2015) y Hernández, et al. (2016), entre otros.

En el marco del proyecto "Investigación para la restauración, la caracterización, zonificación, ordenamiento, restauración y manejo de las ciénagas del departamento del Magdalena”, en 2015 y 2017, entre otras actividades, se desarrollaron estudios batimétricos para la caracterización de los cuerpos de agua objeto del proyecto, dentro de los cuales se encuentra la ciénaga El Chino. Esta ciénaga es un sistema dinámico y sinérgico que se encuentra estrechamente relacionado con la Ciénaga Grande de Santa Marta (CGSM), intercomunicándose a través de una variedad de pequeños caños; sirve como un buffer en los procesos biológicos y fisicoquímicos que se desarrollan en estos cuerpos de agua. A pesar de la importancia de los estudios morfométricos en estos sistemas, no se habían realizado este tipo de estudios en el departamento del Magdalena. El objetivo de este trabajo fue analizar las características batimétricas y morfométricas de la ciénaga El Chino y construir bases científicas elementales en la comprensión de su comportamiento hidromorfológico, a través de la información generada.

\section{MATERIALES Y MÉTODOS}

\section{Área de estudio}

La ciénaga El Chino se localiza entre los $10^{\circ} 51^{\prime} \mathrm{N}$, $74^{\circ} 15^{\prime} \mathrm{O}$ y $10^{\circ} 55^{\prime} \mathrm{N}, 74^{\circ} 19^{\prime} \mathrm{O}$, cerca al piedemonte de la Sierra Nevada de Santa Marta (figura 1). Está aproximadamente a $8 \mathrm{~km}$ al sur de la línea de costa del mar Caribe. Limita al oriente con una zona de planicies fluvio-marinas con pendientes muy bajas, menores al 7 \% (IGAC, 2009); al oeste, con la CGSM; al norte, con la ciénaga de Sevillano con caños más definidos, de mayor profundidad y flujos de agua salobre provenientes del mar Caribe e intercambio más dinámico de sedimentos; y al sur, con los ríos Frío y Sevilla, que le sirven de agua dulce a través de varios caños pequeños. Es un sistema semicerrado, ya que la totalidad de su línea de costa y parte de su superficie está gobernada por abundante manglar. Para los periodos de mediciones del proyecto, del que se obtiene la información del presente trabajo, la temperatura osciló entre $26,5^{\circ} \mathrm{C}$ y $37,1^{\circ} \mathrm{C}$ (promedio: $28,1^{\circ} \mathrm{C}$ ); la humedad relativa estuvo entre el $39 \% \mathrm{y}$ 
el 98,7 \% (promedio: 79,7 \%); el rango de la presión atmosférica fue 1002 a 1017 mbar (promedio: 1010 mbar); finalmente, la radiación solar tuvo valores máximos entre $800 \mathrm{~W} / \mathrm{m}^{2}$ y $1200 \mathrm{~W} / \mathrm{m}^{2}$ con un promedio de $1010 \mathrm{~W} / \mathrm{m}^{2}$. Las precipitaciones en 2015 no superaron los $32 \mathrm{~mm}$. No fue posible tener estos valores para 2017. Los datos de viento mostraron una oscilación semidiurna indicativo de brisas, con direcciones del nor-noroeste, norte y nor-noreste en el día, y valores que no superan los $6 \mathrm{~m} / \mathrm{s}$ en un $9 \%$; en la noche los valores no superan los $4 \mathrm{~m} / \mathrm{s}$ en un $8 \%$ con las direcciones opuestas, principalmente sur-suroeste.

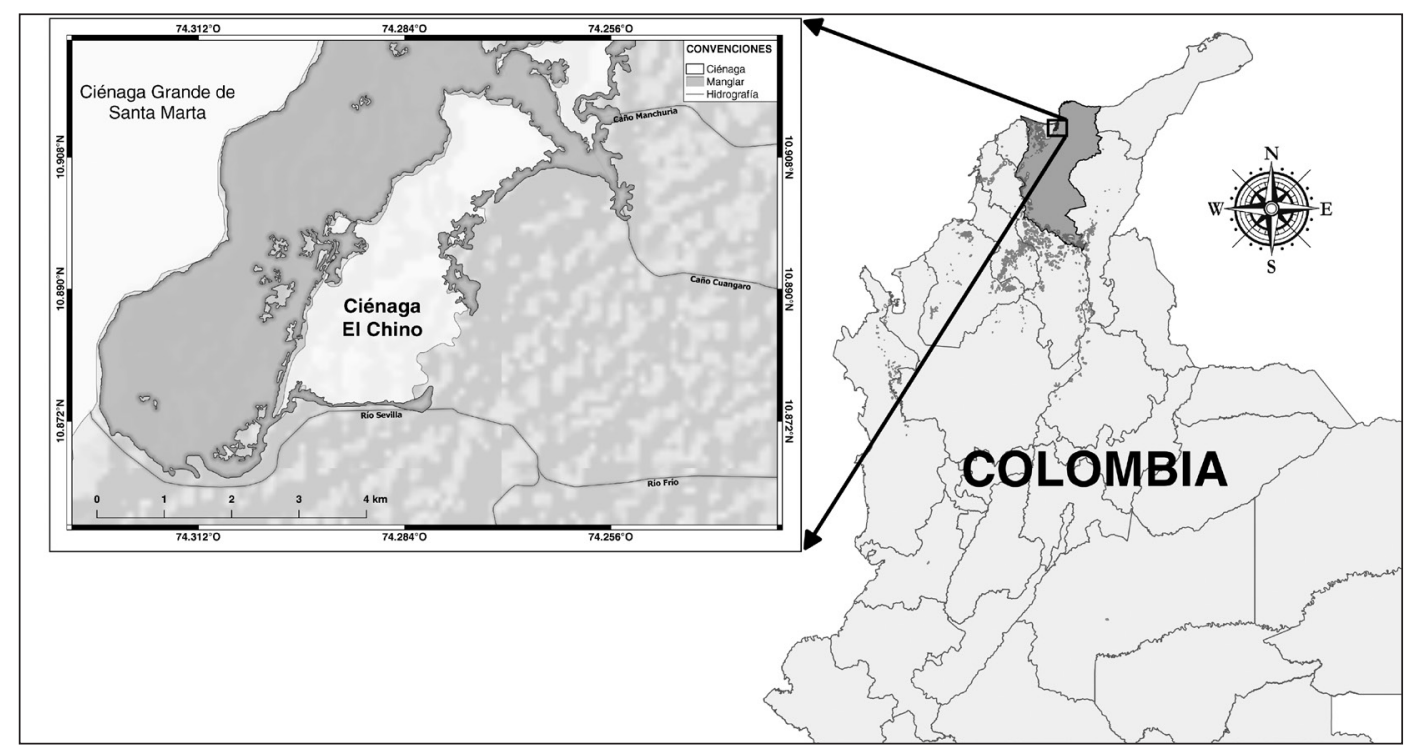

Figura 1. Ubicación espacial de la ciénaga El Chino.

\section{Recolecta y análisis de datos}

Los levantamientos batimétricos fueron realizados en octubre de 2015 y octubre de 2017, con un equipo de sondeo acústico mono haz ó monohaz (Ecosonda Garmin GPSMAP521S), guiado con GPS Garmin Gpsmap 64s, y sensor de nivel para la corrección de marea, siguiendo las normas de la Organización Hidrográfica Internacional para los levantamientos hidrográficos (Bureau Hidrográfico Internacional, 2008), con transectos (planificados con anterioridad e insertados al equipo) espaciados aproximadamente de $100 \mathrm{~m}$.

Se utilizó el software de análisis geocientífico SAGA GIS (Conrad et al., 2015) para hacer la interpolación de los datos de profundidad de la herramienta (module multilevel B-Spiline interpolation), el cual es un algoritmo de interpolación espacial de datos dispersos, propuesto por Lee et al. (1997) para estimar el volumen y el área superficial, construir las curvas hipsométricas y determinar las relaciones de profundidad-área-volumen. El cálculo de los diferentes parámetros morfométricos se realizó siguiendo la metodología de Hakason (1981) y las recomendaciones de Roldán y Ramírez (2008) y Wetzel y Likens (2000). La base de datos obtenida del anterior procedimiento se mapeó en el software Quantum GIS (QGIS Development Team, 2009). Los valores de perímetro se extrajeron del procesamiento de una imagen satelital, cercana al día de sondeo, descargada del servicio geológico de EE.UU. (United States Geological Survey, 2015).

\section{RESULTADOS}

El mapa batimétrico de la ciénaga El Chino evidenció que es un cuerpo de agua somero, que en 2015 (figura 2a) estuvo afectado por las condiciones océanoatmosféricas de El Niño, con profundidades que no superaron los 0,7 $\mathrm{m}$ en algunas partes de la cuenca; sin embargo, en la salida de los caños hacia la ciénaga se encontraron profundidades entre 0,8 y $2 \mathrm{~m}$. En 2017 (Figura 2b) la profundidad promedio fue de $0,77 \mathrm{~m}$ y se observó la influencia de la descargas de los ríos Frío y Sevilla, al igual que el intercambio de flujos con la Ciénaga Grande de Santa Marta y la ciénaga de Sevillano, debido al periodo de lluvias correspondiente al comportamiento de la precipitación en 2017 para esta zona. En igual sentido, se evidenció la redistribución 
de sedimentos en toda la cuenca, dado que el patrón de la distribución de las profundidades cambió entre las épocas comparadas. La consecuencia de las precipitaciones también se ve en el volumen que para el 2017 tuvo un incremento aproximado del $22 \%$, en comparación con el 2015 ( tabla 1). El hecho de que la ciénaga El Chino esté semicerrada por el mangle, ocasiona que su área superficial se vea poco afectada, de allí que los valores sean disímiles en pocos metros cuadrados durante las dos épocas.

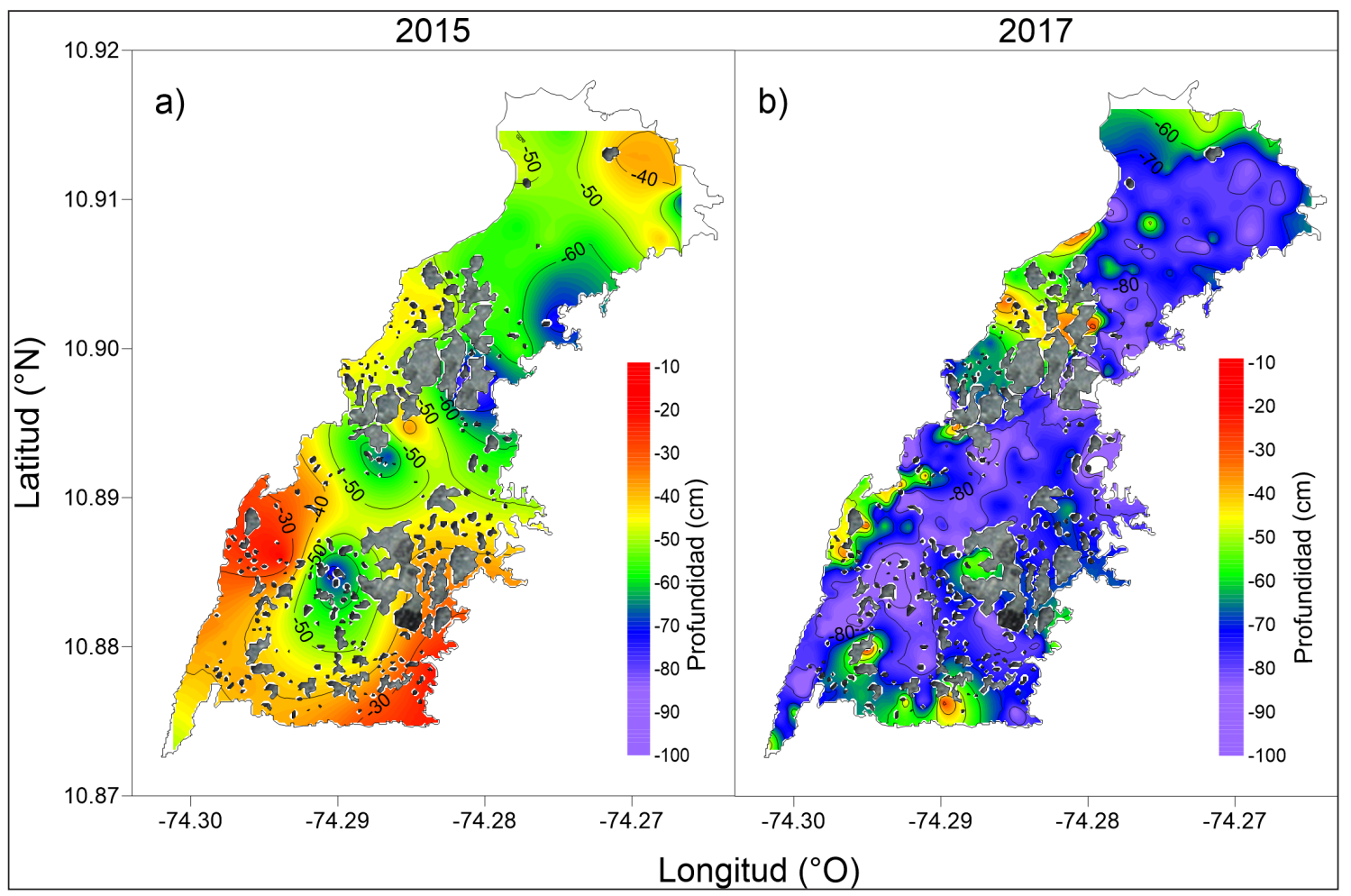

Figura 2. a) Mapa batimétrico de la ciénaga El Chino para el 2015; b) Mapa batimétrico de la ciénaga El Chino para el 2017.

Los parámetros morfométricos de la ciénaga El Chino mostraron una longitud máxima de 5,89 km, orientados en la dirección sur-suroeste (SSO) - nor-noreste (NNE). $\mathrm{El}$ ancho máximo es de 2,14 km. Al estar compuesta por islotes de mangle, el área efectiva, Fetch efectivo, longitud efectiva y ancho efectivo se ven reducidos. El factor de forma indica que es un cuerpo de agua de forma cóncava. Mayores detalles morfométricos están registrados en la tabla 1.

La curva hipsográfica de profundidad vs. área acumulada evidenció que el 50 \% del área acumulada, en ambos periodos, llegó a profundidades cercanas a los $0,6 \mathrm{~m}$ (figura $3 \mathrm{a}$ y $3 \mathrm{c}$ ), y el $50 \%$ del volumen para una profundidad aproximada de 0,3 $\mathrm{m}$ en 2015 (figura 3b) y de 0,4 m en 2017 (figura 3d). En 2017 el $100 \%$ del área acumulada se alcanzó a los 0,4 m de profundidad.

\section{DISCUSIÓN}

Comparando el volumen con otras ciénagas colombianas como El Guájaro (Atlántico) con $182 \mathrm{Mm}^{3}$, Grande de Santa Marta (Magdalena) $2232 \mathrm{Mm}^{3}$, Cachimbero (Santander) 6,8 $\mathrm{Mm}^{3}$, Ayapel (Córdoba) $400 \mathrm{Mm} 3$ (Montoya Moreno et al., 2011) se puede inferir que la ciénaga El Chino (4,302 Mm3 y 5,248 Mm³) es pequeña, pero mayor que Hoyos de los Bagres (Córdoba) con $0,79 \mathrm{Mm}^{3}$. Se debe resaltar que los levantamientos batimétricos se realizaron en épocas distintas y que pueden cambiar a lo largo del tiempo. Esto es una limitante al comparar los resultados obtenidos entre los diferentes autores. La comparación es solo una referencia puesto que el tamaño de una ciénaga respecto a otra no puede ser tomado como una certeza absoluta, ya que la precisión en estimación del volumen y otros cálculos de 
interés limnológicos posteriores en la ciénaga depende de la variación anual en el nivel del lago, que es probable que ocurra (Wetlands International, 2003, citado por Phil et al., 2003). No obstante, dichos cálculos se tienen en cuenta para dar una idea referente del estado de la ciénaga respecto a otras investigaciones similares.

La ciénaga El Chino presentó una superficie alargada y la formación de abundantes islotes de manglar, que rodeó en su mayoría el perímetro y parte de la superficie de la ciénaga, haciendo muy característica la zona marginal de la ciénaga. Esto se ve reflejado en el índice de desarrollo de línea de costa, superior a la unidad $(2,25)$, que en concordancia con lo expresado por Arredondo-Figueroa et al. (1983), El Chino no presentó forma circular, ni cercana a un elipsoide. Contreras et al. (2014) concluyeron que existe mayor correlación entre el índice de desarrollo de costa con el perímetro y no con la superficie, por tanto, independientemente de su tamaño, la ciénaga puede adoptar varias formas a lo largo del tiempo.

En cuanto al índice de desarrollo de volumen, Cole y Weihe (1983) expresaron que el desarrollo del volumen determina la configuración de la cuenca. Sin embargo, valores de este parámetro superiores a la unidad se dan cuando el volumen es superior al que tendría un cono hipotético cuya base tuviera un área igual a la superficie del cuerpo de agua y su altura fuera idéntica a la profundidad máxima registrada para este (Ramon y Moya 1982). Los valores obtenidos de este parámetro indican la tendencia de esta ciénaga a una forma "U" (parábola elíptica), que según Sperling (1999) es un valor típico para la mayoría de lagos del mundo.

Tabla 1. Variables morfométricas de la ciénaga El Chino.

\begin{tabular}{llrr}
\hline \multirow{2}{*}{ Variable } & Abreviatura & \multicolumn{2}{c}{ Valor } \\
\cline { 2 - 4 } & & $\mathbf{2 0 1 5}$ & $\mathbf{2 0 1 7}$ \\
\hline Longitud máxima (km) & L & 5,89 & 5,89 \\
Dirección de eje mayor & Dir & SSO- & SSO \\
Longitud máxima efectiva (km) & & NNE & NNE \\
Ancho máximo (km) & Le & 1,78 & 1,78 \\
Ancho máximo efectivo (km) & W & 2,14 & 2,14 \\
Relación longitud máxima y Ancho máximo & We & 0,56 & 0,56 \\
Área total (km²) & L:W & 2,75 & 2,75 \\
Ancho medio (km) & a & 8,61 & 8,61 \\
Área superficial (km²) & Wm & 1,46 & 1,46 \\
Perímetro o Longitud de línea de costa (km) & A & 7,65 & 7,65 \\
Índice de desarrollo de línea de costa & LC & 23,5 & 23,5 \\
Fetch efectivo (km) & DLC & 2,25 & 2,25 \\
Profundidad máxima (m) & (F) & 2,76 & 2,76 \\
Volumen (Mm³) & (Z) & 0,66 & 0,96 \\
Profundidad media (m) & V & 4,30 & 5,24 \\
Relación profundidad media /Profundidad máxima & Zm:Z & 0,49 & 0,77 \\
Profundidad relativa & Zr & 0,74 & 0,79 \\
Índice de desarrollo de volumen & DV & 2,24 & 0,03 \\
Factor de forma & Vd & Cóncava & Cóncava \\
Relación longitud máxima / Ancho medio & L:Wm & 4,02 & 4,02 \\
Relación ancho máximo /Ancho medio & W:Wm & 1,46 & 1,46 \\
\hline
\end{tabular}


Arredondo-Figueroa et al. (1983) manifestaron que a medida que evolucionan las cuencas de un lago tendrían un valor de desarrollo de volumen superior a la unidad, indicativo de mayor acumulación de sedimentos en la zona litoral y en el fondo; en consecuencia, tendrían una mayor área de contacto entre el sedimento y el agua. Para la ciénaga El Chino se da en gran medida esta condición, por lo que se presume que ha generado pendientes poco pronunciadas debido a la baja profundidad, lo cual podría ser un indicativo de los procesos de sedimentación que estarían afectando al cuerpo de agua. La relación área-volumen, mayor que uno en ambos periodos de muestreo, indica que se tiene una gran zona litoral y lo somero del cuerpo de agua. El factor de forma indica que la ciénaga es cóncava (C), según la clasificación de Håkason (1981), característico de lagos con alto valor de desarrollo de volumen.

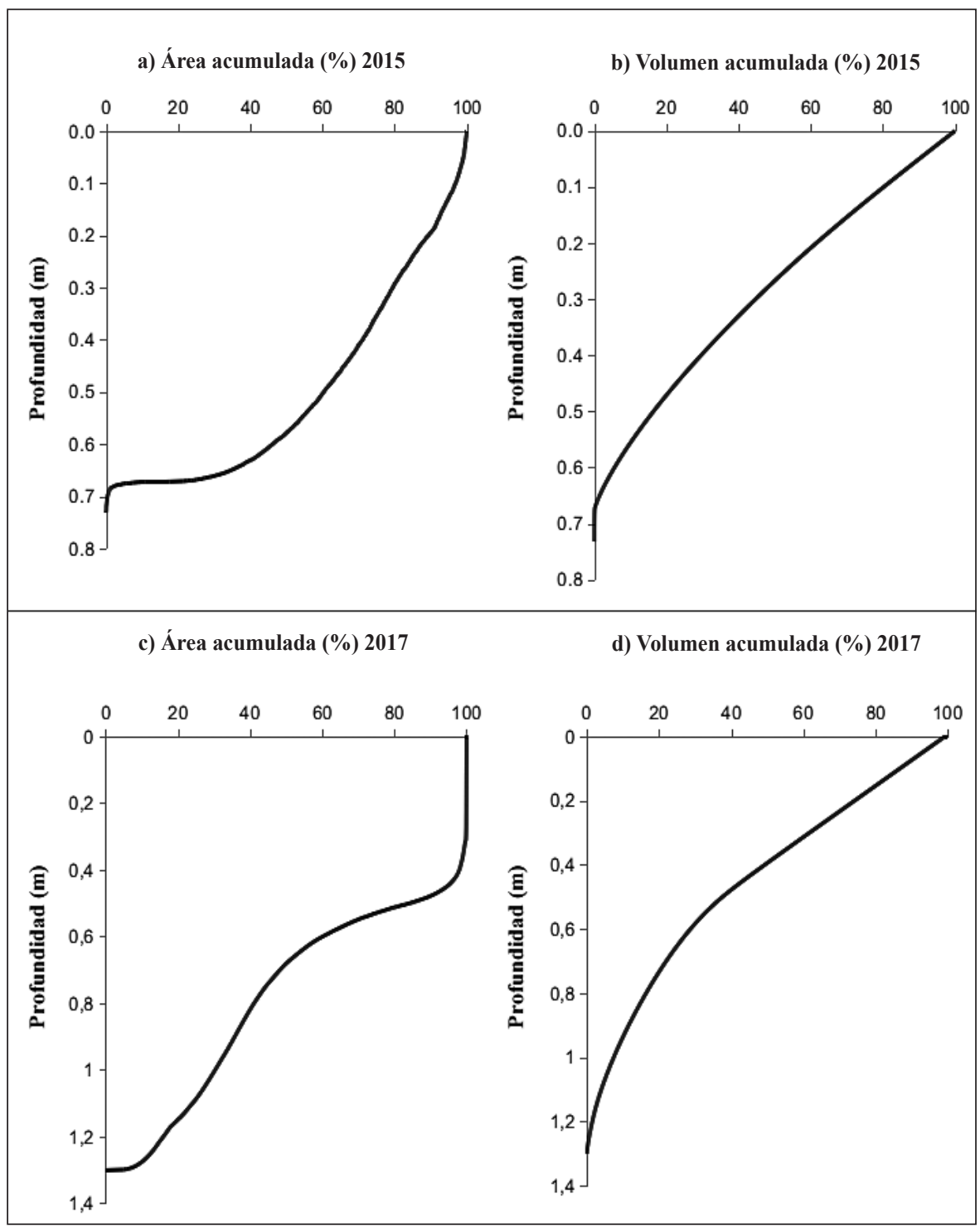

Figura 3. Curvas hipsográficas: a) Área acumulada b) Volumen acumulado de la cienága El Chino para el 2015; c) Área acumulada d) Volumen acumulado de la cienága El Chino para el 2017. 
El bajo valor de la profundidad relativa, sumado a la baja resiliencia térmica de la columna de agua — número de Weddeburn menor que la unidad - (Imberger y Hambling, 1982; Reynolds, 2006) son indicativos de la mayor exposición que tiene la ciénaga El Chino a la influencia del viento, hechos que alteran las condiciones de estabilidad, estratificación, transparencia y nivel de productividad (Hernani y Ramírez, 2002; Montoya Moreno, 2005).

El fetch efectivo (distancia a la cual el viento no puede ser interrumpido) es superior a la longitud máxima efectiva, lo que permite inferir que no se tiene el espacio suficiente para el desarrollo de olas de fondo (swell). Håkanson y Jansson (2002) definieron el cociente $\mathrm{A}^{\frac{1}{2} / 2}$ ${ }^{\mathrm{Zm}}$ como el radio dinámico, el cual enfatiza la dinámica de fondo. Como el valor encontrado en $2015(\sim 4,15)$ es superior a 3,8, cuando se calcule el valor del área erosión + transporte $(\mathrm{AE}+\mathrm{T})$ a partir de las curvas hipsográficas, éste debe corregirse; en 2017 (3,59) no es necesario. Estos autores manifiestan que si los radios dinámicos son superiores a 0,8 implica que los cuerpos de agua estarían sujetos a efectos del oleaje en toda el área, por lo menos una parte del tiempo. Como el área es mayor en comparación con la profundidad promedio, la acción del viento y del oleaje sobre el cuerpo de agua tiene mayor importancia, por lo que los procesos de erosión y de transporte son dominantes en la mayoría de aspectos limnológicos, dependiendo de la energía disponible para mezclar el agua. Por lo anterior, se podría inferir que la ciénaga El Chino es muy inestable, no existen estratos de temperatura y de llegar a existir serían inmediatamente perturbados por el viento, la lluvia u hora del día.

La profundidad media es inferior a la profundidad mediana (segundo cuartil), esto es característico de los lagos con curvas hipsográficas relativas cóncavas (Håkason, 1981). Del mismo modo de la relación profundidad media /profundidad máxima ( $\mathrm{Zm:Z} \mathrm{>}$ $0,4)$ se puede inferir, según Montoya Moreno (2005b), que puede presentar mayores riesgos de colmatación.

Debido a la influencia marina y la desecación por las bajas o nulas precipitaciones ocasionadas por el fenómeno de El Niño en 2015, se encontraron condiciones hipersalinas de hasta tres veces la salinidad media el mar $(91,72)$, lo que pudo favorecer la proliferación de organismos halófitos como la microalga Dunaliella salina Teodoresco, 1905, que le confiere un matiz de colores rojizo al agua, especialmente en la parte sur de la ciénaga. Los cambios de coloración entre la parte norte y sur podrían estar relacionados con diferentes procesos de mezcla y circulación, inducidos por los cinturones de manglar que se desarrollan en el "centro", parte sureste de la ciénaga, que dividen a El Chino en dos cubetas hídricas que se evidencian en el mapa batimétrico (figura 2a). De esta manera, se podría estar debatiendo acerca de un posible fenómeno de "polimixis zonificada" gracias a un buffer natural de manglares que trabaja como una barrera semipermeable en los procesos sinérgicos de la ciénaga.

\section{CONCLUSIONES}

La ciénaga El Chino se puede considerar un sistema lagunar muy somero, con profundidad relativa muy baja y resiliencia térmica bajas, lo que implica que se alteran con facilidad las condiciones de estabilidad, transparencia y nivel de productividad. Es un cuerpo de agua muy inestable, no existen estratos de temperatura y de llegar a existir serían inmediatamente perturbados por el viento, la lluvia o la hora del día. Presenta un índice de desarrollo de volumen alto, lo que se ve reflejado en los elevados procesos de sedimentación, una forma cóncava semejante a un elipsoide en la que existe la formación de dos pequeñas cuencas. La acción del viento se ve frenada por la espesa vegetación de manglar, que forma una barrera de islotes en algunas zonas, lo que podría inducir la zonificación de parámetros fisicoquímicos y biológicos en la ciénaga. Las características morfométricas se pueden ver modificadas debido a los periodos de lluvia, que tienen la facultad desencadenar pulsos de inundación y modificar dichos parámetros. Sin embargo, los dos periodos de estudio mostraron un sistema con muchos parámetros similares y la época de lluvias en 2017 no mejora ostensiblemente algunos parámetros que son indicativos de la salud del mismo.

Por otro lado, las actividades antrópicas podrían estar generando impactos ambientales negativos, mediante el desvío y taponamiento de caños, que a su vez afectan la hidráulica de la ciénaga y causan sinergismo anómalo en los ecológicos naturales. Sin embargo, se necesitan estudios adicionales para comprobar esta afirmación, modelar la calidad del agua y proponer alternativas de mejoramiento integral del sistema.

\section{AGRADECIMIENTOS}

El presente estudio fue realizado gracias al proyecto "Investigación para la restauración, caracterización, zonificación, ordenamiento, restauración y manejo de las ciénagas del departamento del Magdalena” BPIN: 2013000100017, financiado bajo el convenio 
de cooperación 096/2013 entre la Fundación para la Participación, Capacitación y la Investigación Social "FUPARCIS" y la Gobernación del Magdalena, con recursos del Fondo de Ciencia y Tecnología del Sistema General de Regalías. Agradecimiento especial para los componentes SIG, Biológico e Hidrodinámico, de la fundación FUPARCIS, por la información prestada. Agradecimientos especiales a los ingenieros ambientales y sanitarios Andrea Manrique Cantillo y Arturo Rozo Celemín por su ayuda en la obtención de los datos en campo, y al M.Sc Gustavo Manjarrés García por todo su apoyo y magnífico entusiasmo por la investigación.

\section{REFERENCIAS}

Aldama, G.R., Ponce, Palafox, J., Cruz, J., Hernández, D., Díaz, J. y Arredondo-Figueroa, J. 2013. Biociencias 2(2): 83-88.

Benjumea Hoyos, C., Wills Toro, Á. y Aguirre, N. 2010. Principales aspectos morfométricos de la ciénaga de Cachimbero, Santander - Colombia. Gestión y Ambiente 11(2): I-VIII.

Bureau Hidrográfico Internacional. 2008. Normas de la OHI para los levantamientos hidrográficos. Publicación Especial No. 44. 5ª Edición, Mónaco.

Castro Rodríguez, S.Y. 2012. Análisis florístico y fitogeográfico de ambientes asociados al complejo de ciénagas de Zapatosa (Cesar) en el Caribe colombiano. Tesis de Maestría, Universidad Nacional de Colombia, Bogotá, Colombia.

Cole, G. y Weihe, P. 1983. Textbook of limnology. Waveland Press, Inc. Dallas TX.

Conrad, O., Bechtel, B., Bock, M., Dietrich, H., Fischer, E., Gerlitz, L. y Böhner, J. 2015. System for Automated Geoscientific Analyses (SAGA) Geosci. Model Dev (Versión v. 2.1.4).

Contreras, F., Ojeda, E. y Contreras, S. 2014. Aplicación de la línea de costa en el estudio morfométrico de las lagunas de las lomadas arenosas de Corrientes, Argentina. Contribuciones Científicas GÆA 26: 65-78.

Figueroa Arredondo, J., Borrego Enríquez, L., Castillo Domínguez, R.M. y Valladolid Laredo, M.A. 1983. Batimetría y morfometría de los lagos "Maars" de la Cuenca Oriental, Puebla, México. Biótica 8: 37-47.

Gómez Rivera, M. 2008. Variación espaciotemporal de macroinvertebrados asociados a las raíces de Eichhornia crassipes (Mart) Solms- Laubach 1883, en tres ciénagas del Caribe colombiano. Trabajo de grado, Universidad Jorge Tadeo Lozano, Bogotá, Colombia.

Guiral, J. y Pérez, E. 1980. Based on a work plan and a study for projects on protection of humid areas. Boletín de la Estación Central de Ecología 9: 19-26.

Håkanson, L. 2005. The importance of Lake Morphometry and catchment characteristics in limnology - ranking based on statistical analyses. Hydrobiologia 541(1): 117-137.

Håkason, L. 1981. A manual of Lake Morphometry. Springer-Verlag-Berlin Heidelberg, New York.

Hernani, T. y Ramírez, R. 2002. Aspectos morfométricos y teóricos de un embalse tropical de alta montaña: represa La Fe, Retiro, Colombia. Caldasia 26: 511-518.

Håkanson, L. y Jansson, M. 2002. Principles of Lake Sedimentology. Blackburn Press, Nueva Jersey.

Hernández-Atilano, E., Aguirre, N.J., Palacio, J.A. y Ramírez-Restrepo, J.J. 2008. Variación espacio-temporal de la asociación fitoplanctónica en diferentes momentos del pulso hidrológico en la ciénaga de Ayapel (Córdoba), Colombia. Actualidades Biológicas 30(88): 67-81.

Hernández, J.L., Guzmán-Soto, C.J., Tamaris-Turizo, C.E. 2016. Macroinvertebrados acuáticos de la ciénaga de Sahaya y en tres de sus afluentes (Cesar, Colombia). Intropica 11:11-20.

Hutchinson, G. 1957. A treatise on Limnology. Geography, Physics and Chemistry. John Wiley and Sons, Nueva York.

Imberger, J. y Hambling, P.F. 1982. Dynamics of lakes, reservoirs and cooling ponds. Annual Review of Fluid Mechanichs 14: 153-87.

IGAC. 2009. Estudio general de suelos y zonificación de tierras. Departamento del Magdalena. escala 1:100000 (Vol. 1). Instituto Geográfico "Agustín Codazzi." Subdirección Agrológica, Bogotá.

Jiménez-Segura, L.F., Carvajal-Quintero, J.D. y Aguirre, N. 2010. Las ciénagas como hábitat para los peces: estudio de caso en la ciénaga de Ayapel (Córdoba). Actualidades biológicas 32: 53-64

Lee, S., Wolberg, G. y Shin, S.Y. 1997. Scattered Data Interpolation with Multilevel B-Splines. IEEE Transactions on Visualization and Computer Graphics 3(3): 228-244. 
Molano-Rendón, F., Morales-Castaño, I. y SerratoHurtado, C. 2008. Clasificación y hábitats de Gerridae (Heteroptera - Gerromorpha) en Colombia. Acta Biológica Colombiana 13(2): 41-60.

Montoya Moreno, Y. 2005. Caracterización morfométrica básica de tres lagos someros en el municipio de El Carmen de Viboral (Antioquia), Colombia. Revista Actualidades Biológicas 27: 79-86.

Montoya Moreno, Y. 2008. Caracterización morfométrica de un sistema fluviolacustre tropical, Antioquia, Colombia. Caldasia 30(2): 413-420.

Montoya Moreno, Y. y Aguirre Ramírez, N. 2009. Cambios nictemerales de variables físicas y químicas en la Ciénaga de Paticos, complejo cenagoso de Ayapel, Colombia. Revista de Biología Tropical 57(3): 635-646.

Montoya Moreno, Y., Vélez, F. y Aguirre Ramírez, N. 2011. Características morfométricas de un lago de plano inundable tropical (ciénaga Hoyo Los Bagres, Colombia). Revista de la facultad de ingeniería 59: 203-214.

Neuman, J. 1959. Maximum depth and average depth of lakes. Journal of the Fisheries Research Board of Canada 16(6): 923-927.

Phil, H., Rosalind R. y Kenneth, M. 2003. Bathymetry of Lake Bogoria, Kenya. Nature Kenya/East African Natural History Society 92: 107-117.

QGIS Development Team. 2016. QGIS Geographic Information System. Open Source Geospatial Foundation. http://qgis.osgeo.org. Consultado: 26 de diciembre 2017. Ramon, G. y Moya, G. 1982. Características Morfológicas y morfométricas de los embalses de Cuber y Gorc Blau (Mallorca). Bulletin de la Société d'histoire naturelle de Toulouse 26: 145-150.

Restrepo, C., Toro, M. y Aguirre Ramírez, N. 2006. Aproximación a la dinámica del transporte del nitrógeno y del fósforo en la ciénaga de Ayapel. Avances en Recursos Hidráulicos 13: 7-22.

Reynolds, C.S. 2006. The ecology of phytoplankton. Ecology, Biodiversity and Conservation. Cambridge University Press, Cambridge.

Rico, E., González, M. y Montes, C. 1995. Batimetría y análisis morfométrico del lago de Arreo (N. España). Limnética 11: 55-58.

Rivera Rondón, C., Zapata, A. y Donato Rondon, J. 2010. Estudio morfométrico del lago Guatavita (Colombia). Acta Biológica Colombiana 15(3): 131-144.

Roldan, G. y Ramírez, J. 2008. Fundamentos de limnología neotropical. Segunda edición, Editorial de la Universidad de Antioquia, Medellín.

Rúa-García, Gustavo. 2015. Macroinvertebrados acuáticos asociados a raíces de Eichhornia crassipes (Mart) Solms, en la ciénaga de Zapayán, Magdalena-Colombia. Intropica 10: 52-59.

Sperling, E. 1999. Morfología de lagos e represas. Departamento de Engenharia Sanitária e Ambiental (DESA/ UFMG), Belo Horizonte.

United States Geological Survey. 2015. Earthexplorer. http://earthexplorer.usgs.gov/. Consultado: 15 de enero 2018.

Wetzel, R. y Likens, G. 1991. Limnological analyses. Second edition, Springer-Verlag, Nueva York.

Wetzel, R. y Likens, G. 2000. Limnological analyses. Third edition, Springer-Verlag, Nueva York.

Zapata Gaviria, I. 2005. Dinámica hidrológica en la ciénaga de Ayapel. Modelación del balance hídrico y simulación hidrodinámica. Tesis de Maestría, Universidad Nacional de Colombia, Medellín, Colombia.

Fecha de recepción: 24/01/2018

Fecha de aceptación: 16/03/2018

Publicado en línea: 01/04/2018

Para citar este artículo: Rodríguez Curcio J.J., Linero-Cueto J. y Barros Ortiz L.J. 2018. Caracterización morfométrica de una laguna costera Neotropical (ciénaga El Chino, Magdalena- Colombia). Intropica 13(1): 21-29. 